\title{
On the Uniform and Simultaneous Approximations of Functions
}

\author{
Mansour Alyazidi \\ Department of Mathematics, King Saud University, Riyadh, Saudi Arabia \\ Email: yazidi@ksu.edu.sa
}

How to cite this paper: Alyazidi, M. (2021) On the Uniform and Simultaneous Approximations of Functions. Advances in Pure Ma thematics, 11, 785-790.

https://doi.org/10.4236/apm.2021.1110052

Received: September 9, 2021

Accepted: October 10, 2021

Published: October 13, 2021

Copyright (c) 2021 by author(s) and Scientific Research Publishing Inc. This work is licensed under the Creative Commons Attribution International License (CC BY 4.0)

http://creativecommons.org/licenses/by/4.0/

\begin{abstract}
We consider the relation between the simultaneous approximation of two functions and the uniform approximation to one of these functions. In particular, $F_{1}$ and $F_{2}$ are continuous functions on a closed interval $[a, b], S$ is an $n$-dimensional Chebyshev subspace of $C[a, b]$ and $s_{1}^{*} \& s_{2}^{*}$ are the best uniform approximations to $F_{1}$ and $F_{2}$ from $S$ respectively. The characterization of the best approximation solution is used to show that, under some restrictions on the point set of alternations of $F_{1}-s_{1}^{*}$ and $F_{2}-s_{2}^{*}, s_{1}^{*}$ or $s_{2}^{*}$ is also a best $A(1)$ simultaneous approximation to $F_{1}$ and $F_{2}$ from $S$ with $F_{1} \geq F_{2}$ and $n=2$.
\end{abstract}

\section{Keywords}

Simultaneous Approximation, Uniform Approximation, Relation, Straddle Points, Alternation

\section{Introduction}

The interest in the simultaneous approximation started long ago [1] [2] [3] [4]. This paper concerned with the relation between the simultaneous approximation and the uniform approximation. The setting is as follows. Let $C[a, b]$ be the set of all real-valued continuous functions defined on the closed interval $[a, b]$ with the uniform norm $\|$.$\| .$

For $f \in C[a, b]$,

$$
\|f\|=\max \{|f(x)|, x \in[a, b]\} .
$$

The norms $\|F\|_{A(p)}, 1 \leq p \leq \infty$, on $E=C[a, b] \times C[a, b]$ are defined as follows: 
For $F=\left(F_{1}, F_{2}\right) \in E$

$$
\begin{gathered}
\|F\|_{A(\infty)}=\max \left\{F_{1}, F_{2}\right\} \\
\|F\|_{A(p)}=\left[\left\|F_{1}\right\|^{p}+\left\|F_{2}\right\|^{p}\right]^{\frac{1}{p}}, 1 \leq p<\infty .
\end{gathered}
$$

Now if $S$ is an $n$-dimensional subspace of $C[a, b]$, then $U=\{(s, s): s \in S\}$ is an $n$-dimensional subspace of $E$ and there exist $u^{*}=\left(s^{*}, s^{*}\right)$ and $v^{*}=\left(t^{*}, t^{*}\right)$ where $s^{*}, t^{*} \in S$ such that:

$$
\begin{aligned}
\left\|F-u^{*}\right\|_{A(\infty)} & =\inf _{u \in U}\|F-u\|_{A(\infty)} \\
& =\inf _{s \in S} \max \left\{\left\|F_{1}-s\right\|,\left\|F_{2}-s\right\|\right\} \\
& =\left\|F_{k}-s^{*}\right\|, \quad k=1 \text { or } 2 .
\end{aligned}
$$

Such $s^{*}$ is called a best $A(\infty)$ simultaneous approximation to $F=\left(F_{1}, F_{2}\right)$ from $S$. The set of all best $A(\infty)$ simultaneous approximations to $F$ from $S$ will be denoted by $P_{S}(F, \infty)$.

For $1 \leq p<\infty$,

$$
\begin{aligned}
\left\|F-v^{*}\right\|_{A(p)} & =\inf _{u \in U}\|F-u\|_{A(p)} \\
& =\inf _{s \in S}\left\{\left[\left\|F_{1}-s\right\|^{p}+\left\|F_{2}-s\right\|^{p}\right]^{\frac{1}{p}}\right\} \\
& =\left[\left\|F_{1}-t^{*}\right\|^{p}+\left\|F_{2}-t^{*}\right\|^{p}\right]^{\frac{1}{p}} .
\end{aligned}
$$

$t^{*}$ is called a best $A(p)$ simultaneous approximation to $F=\left(F_{1}, F_{2}\right)$ from $S$. The set $P_{S}(F, p)$ denotes the set of all best $A(p)$ simultaneous approximation to $F$ from $S$. And $P_{S}\left(F_{k}\right)$ is the set of all best uniform approximation to $F_{k}$ from $S, k \in\{1,2\}$.

We are interested in the relation between the simultaneous approximation and the uniform approximation; in section two, we will show under certain conditions, that if $s_{k}^{*} \in P_{S}\left(F_{k}\right)$ then $s_{k}^{*} \in P_{S}(F, 1), k \in\{1,2\}$.

Definition 1 A point $t \in[a, b]$ is called a straddle point for two functions $f$ and $g$ in $C[a, b]$ if there exists $\sigma= \pm 1$ such that

$$
\|f\|=\sigma f(t),\|g\|=-\sigma g(t) .
$$

Definition 2 The functions $f$ and $g \in C[a, b]$ are said to have $d$ alternations on $[a, b]$ if there exists $d+1$ distinct points $x_{1}<\cdots<x_{d+1}$ in $[a, b]$ such that for some $\sigma= \pm 1$,

$$
\begin{gathered}
f\left(x_{i}\right)=\sigma\|f\|, \text { if } i \text { is odd } \\
g\left(x_{i}\right)=-\sigma\|g\|, \text { if } i \text { is even }
\end{gathered}
$$

or

$$
\begin{gathered}
g\left(x_{i}\right)=\sigma\|g\|, \text { if } i \text { is odd } \\
f\left(x_{i}\right)=-\sigma\|f\|, \text { if } i \text { is even }
\end{gathered}
$$


We follow [5] [6] [7] [8] for the notations and the terminology of this section which will be used throughout this paper. The uniform approximation theory can be found in [9] [10]. Theorems 1 and 2 of this section and the remark thereafter which are needed for our analysis are direct consequences of theorems 1 and 3 of [6].

Theorem 1 Let $S$ be an $n$-dimensional subspace of $C[a, b]$ which contains a nonzero constant, $F=\left(F_{1}, F_{2}\right) \in E$ then:

(a) $s^{*} \in P_{S}(F, 1)$ if and only if there exists subsets $X_{1}=\left\{x_{i}, i \in I_{1}\right\}$, $X_{2}=\left\{x_{i}, i \in I_{2}\right\}$ of $[a, b]$ and positive numbers $\lambda_{i}, i \in I_{1}, \mu_{i} \in I_{2}$ with

$$
\sum_{i \in I_{1}} \lambda_{i}=\sum_{i \in I_{2}} \mu_{i}=1
$$

such that

$$
\begin{gathered}
\theta_{i}\left(F_{1}\left(x_{i}\right)-s^{*}\left(x_{i}\right)\right)=\left\|F_{1}-s^{*}\right\|, \quad i \in I_{1}, \\
\theta_{i}\left(F_{2}\left(x_{i}\right)-s^{*}\left(x_{i}\right)\right)=\left\|F_{2}-s^{*}\right\|, \quad i \in I_{2}, \\
\sum_{i \in I_{1}} \theta_{i} \lambda_{i} s\left(x_{i}\right)+\sum_{i \in I_{2}} \theta_{i} \mu_{i} s\left(x_{i}\right)=0 \quad \forall s \in S, \\
\theta_{i}= \pm 1 .
\end{gathered}
$$

(b) If $s^{*} \in P_{s}(F, 1)$ with $\left\|F_{1}-s^{*}\right\|=\left\|F_{2}-s^{*}\right\|$ then $s^{*} \in P_{S}(F, p)$ for all $p$,

$$
1<p \leq \infty \text {. }
$$

Theorem 2 Let $S$ be an $n$-dimensional Haar subspace of $C[a, b]$, if $F_{1} \geq F_{2}$ on $[a, b]$ then $s^{*} \in P_{s}(F, \infty)$ if and only if $F_{1}-s^{*} \& F_{2}-s^{*}$ have a straddle point or $n$ alternations on $[a, b]$ with $\left\|F_{1}-s^{*}\right\|=\left\|F_{2}-s^{*}\right\|$. Furthermore, if $F_{1}-s^{*} \& F_{2}-s^{*}$ have $n$ alternations on $[a, b]$ then $s^{*}$ is unique.

Remark If $t \in[a, b]$ is a straddle point for $F_{1}-s^{*} \& F_{2}-s^{*}, F_{1} \geq F_{2}$ on $[a, b]$ then

$$
\left(F_{1}-F_{2}\right)(t)=\left(F_{1}-s^{*}\right)(t)+\left(F_{2}-s^{*}\right)(t)=\left\|F_{1}-s^{*}\right\|+\left\|F_{2}-s^{*}\right\| \geq\left\|F_{1}-F_{2}\right\| .
$$

This implies that $\left(F_{1}-F_{2}\right)(t)=\left\|F_{1}-F_{2}\right\|$ and

$$
\left\|F_{1}-s^{*}\right\|+\left\|F_{2}-s^{*}\right\|=\left\|F_{1}-F_{2}\right\| \leq\left\|F_{1}-s\right\|+\left\|F_{2}-s\right\| \quad \forall s \in S .
$$

Hence $s^{*} \in P_{S}(F, 1)$.

\section{The Main Result}

Theorem 3 Let $s_{k}^{*} \in P_{S}\left(F_{k}\right)$, where $F_{k} \in C[a, b], k \in\{1,2\}, \quad F_{1} \geq F_{2}$ on $[a, b], \quad F=\left(F_{1}, F_{2}\right)$ and $S$ is a 2-dimensional Chebyshev subspace of $C[a, b]$ containing a nonzero constant function. And let $X=\left\{a=x_{1}<x_{2}<x_{3}=b\right\}$ be the alternating set for $F_{1}-s_{1}^{*}, \quad Y=\left\{a=y_{1}<y_{2}<y_{3}=b\right\}$ be the alternating set for $F_{2}-s_{2}^{*}$.

(i) If $\left(F_{1}\left(x_{1}\right)-s_{1}^{*}\left(x_{1}\right)\right)=\left\|F_{1}-s_{1}^{*}\right\|$ and $\left(F_{2}\left(y_{1}\right)-s_{2}^{*}\left(y_{1}\right)\right)=\left\|F_{2}-s_{2}^{*}\right\|$, then $s_{1}^{*} \in P_{S}(F, 1)$.

(ii) If $\left(F_{1}\left(x_{1}\right)-s_{1}^{*}\left(x_{1}\right)\right)=-\left\|F_{1}-s_{1}^{*}\right\|$ and $\left(F_{2}\left(y_{1}\right)-s_{2}^{*}\left(y_{1}\right)\right)=-\left\|F_{2}-s_{2}^{*}\right\|$, then $s_{2}^{*} \in P_{S}(F, 1)$. 


\section{Proof}

(i) suppose that $\left(F_{1}\left(x_{1}\right)-s_{1}^{*}\left(x_{1}\right)\right)=\left\|F_{1}-s_{1}^{*}\right\|$ and $\left(F_{2}\left(y_{1}\right)-s_{2}^{*}\left(y_{1}\right)\right)=\left\|F_{2}-s_{2}^{*}\right\|$, since $-F_{2} \geq-F_{1}$ then

$$
\left(s_{1}^{*}\left(x_{2}\right)-F_{2}\left(x_{2}\right)\right) \geq\left(s_{1}^{*}\left(x_{2}\right)-F_{1}\left(x_{2}\right)\right)=\left\|F_{1}-s_{1}^{*}\right\|
$$

and if $x \in[a, b]$ is such that $\left(F_{2}-s_{1}^{*}\right)(x) \geq 0$, then

$$
\left\|F_{1}-s_{1}^{*}\right\| \geq\left(F_{1}-s_{1}^{*}\right)(x) \geq\left(F_{2}-s_{1}^{*}\right)(x) \geq 0 \text {. }
$$

Hence there exists a $\gamma \in[a, b]$ such that

$$
\left\|F_{2}-s_{1}^{*}\right\|=-\left(F_{2}-s_{1}^{*}\right)(\gamma) \text {. }
$$

If $\gamma=a$ or $\gamma=b$ then $\gamma$ is a straddle point for $F_{1}-s_{1}^{*} \& F_{2}-s_{1}^{*}$ which implies that $s_{1}^{*} \in P_{S}(F, 1)$.

If $a<\gamma<b$ then taking $x_{1}=z_{1}, \gamma=z_{2}, x_{3}=z_{3}$ we have:

$$
\begin{gathered}
\left(F_{1}-s_{1}^{*}\right)\left(z_{1}\right)=\left(F_{1}-s_{1}^{*}\right)\left(z_{3}\right)=\left\|F_{1}-s_{1}^{*}\right\|, \\
-\left(F_{2}-s_{1}^{*}\right)\left(z_{2}\right)=\left\|F_{2}-s_{1}^{*}\right\|, \\
a \leq z_{1}<z_{2}<z_{3} \leq b .
\end{gathered}
$$

Now, since $S$ is a Chebyshev subspace of dimension 2, there exists $\mu_{i}>0, i \in\{1,2,3\}$ such that

$$
\mu_{1} s\left(z_{1}\right)-\mu_{2} s\left(z_{2}\right)+\mu_{3} s\left(z_{3}\right)=0 \quad \forall s \in S
$$

because $1 \in S, \mu_{2}=\mu_{1}+\mu_{3}$ and setting $\omega_{i}=\frac{\mu_{i}}{\mu_{2}}, i \in\{1,2,3\}$ we have $\omega_{1} s\left(z_{1}\right)-\omega_{2} s\left(z_{2}\right)+\omega_{3} s\left(z_{3}\right)=0 \quad \forall s \in S \quad$ where $\quad \omega_{2}=\omega_{1}+\omega_{3}=1 \quad$ and from theorem $1 s_{1}^{*} \in P_{S}(F, 1)$.

ii) If $\left(F_{1}\left(x_{1}\right)-s_{1}^{*}\left(x_{1}\right)\right)=-\left\|F_{1}-s_{1}^{*}\right\|$ and $\left(F_{2}\left(y_{1}\right)-s_{2}^{*}\left(y_{1}\right)\right)=-\left\|F_{2}-s_{2}^{*}\right\|$, since $F_{1} \geq F_{2}$ then

$$
\left(F_{1}\left(y_{2}\right)-s_{2}^{*}\left(y_{2}\right)\right) \geq\left(F_{2}\left(y_{2}\right)-s_{2}^{*}\left(y_{2}\right)\right)=\left\|F_{2}-s_{2}^{*}\right\|
$$

and if $x \in[a, b]$ is such that $\left(s_{2}^{*}-F_{1}\right)(x) \geq 0$, then

$$
\left\|F_{2}-s_{2}^{*}\right\| \geq\left(s_{2}^{*}-F_{2}\right)(x) \geq\left(s_{2}^{*}-F_{1}\right)(x) \geq 0 .
$$

Hence there exists a $\gamma \in[a, b]$ such that

$$
\left\|F_{1}-s_{2}^{*}\right\|=\left(F_{1}-s_{2}^{*}\right)(\gamma) \text {. }
$$

If $\gamma=a$ or $\gamma=b$ then $\gamma$ is a straddle point for $F_{1}-s_{2}^{*} \& F_{2}-s_{2}^{*}$ which implies that $s_{2}^{*} \in P_{S}(F, 1)$.

If $a<\gamma<b$ then taking $y_{1}=z_{1}, \gamma=z_{2}, y_{3}=z_{3}$ we have:

$$
\begin{gathered}
\left(F_{2}-s_{2}^{*}\right)\left(z_{1}\right)=\left(F_{2}-s_{2}^{*}\right)\left(z_{3}\right)=-\left\|F_{1}-s_{1}^{*}\right\|, \\
\left(F_{1}-s_{2}^{*}\right)\left(z_{2}\right)=\left\|F_{1}-s_{2}^{*}\right\|, \\
a \leq z_{1}<z_{2}<z_{3} \leq b .
\end{gathered}
$$

Now, since $S$ is a Chebyshev subspace of dimension 2, there exists 
$\mu_{i}>0, i \in\{1,2,3\}$ such that

$$
-\mu_{1} s\left(z_{1}\right)+\mu_{2} s\left(z_{2}\right)-\mu_{3} s\left(z_{3}\right)=0 \quad \forall s \in S
$$

because $1 \in S, \mu_{2}=\mu_{1}+\mu_{3}$ and setting $\omega_{i}=\frac{\mu_{i}}{\mu_{2}}, i \in\{1,2,3\}$ we have $-\omega_{1} s\left(z_{1}\right)+\omega_{2} s\left(z_{2}\right)-\omega_{3} s\left(z_{3}\right)=0 \quad \forall s \in S \quad$ where $\omega_{2}=\omega_{1}+\omega_{3}=1$ and from theorem $1 s_{2}^{*} \in P_{S}(F, 1)$ and the theorem is proved.

The following example shows that conditions (i) \& (ii) in theorem 3 are necessary conditions.

Example $1 S=\operatorname{span}\{1, x\}$ is a Chebyshev subspace of $C[0,1]$ and $s_{1}^{*}=\frac{1}{8}+x$ is the best uniform approximation to $F_{1}=\sqrt{x}, s_{2}^{*}=\frac{-1}{8}+x$ is the best uniform approximation to $F_{2}=x^{2}, F_{1} \geq F_{2}$ on $[0,1], s_{1}^{*} \notin P_{S}(F, 1)$ and $s_{2}^{*} \notin P_{S}(F, 1)$.

It is possible, under the assumptions of theorem 3 that both $s_{1}^{*}$ and $s_{2}^{*}$ belong to the set of best $A(1)$ simultaneous approximation as illustrated in the following example

Example $2 S=\operatorname{span}\{1, x\}$ is a Chebyshev subspace of $C[0,1]$ and $s_{1}^{*}=\frac{-1}{8}+x$ is the best uniform approximation to $F_{1}=x^{2}, s_{2}^{*}=\frac{-1}{3 \sqrt{3}}+x$ is the best uniform approximation to $F_{2}=x^{3}, F_{1} \geq F_{2}$ on $[0,1]$.

$s_{1}^{*}, s_{2}^{*} \in P_{S}(F, 1)$. Furthermore $s_{2}^{*}=\frac{-1}{3 \sqrt{3}}+x$ is the unique best $A(\infty)$ simultaneous approximation to $F=\left(F_{1}, F_{2}\right)$ from $S$.

\section{Conflicts of Interest}

The author declares no conflicts of interest regarding the publication of this paper.

\section{References}

[1] Dunham, C.B. (1967) Simultaneous Chebyshev Approximation of Two Functions on an Interval. Proceedings of the AMS, 18, 472-477. https://doi.org/10.1090/S0002-9939-1967-0212463-6

[2] Watson, G.A. (1993) A Characterization of Best Simultaneous Approximation. Journal of Approximation Theory, 75, 175-182. https://doi.org/10.1006/jath.1993.1097

[3] Huotari, R. and Shi, J. (1995) Simultaneous Approximation from Convex Sets. Computers \& Mathematics with Applications, 30, 197-206. https://doi.org/10.1016/0898-1221(95)00097-6

[4] Pinkus, A. (1997) Uniqueness in Vector-Valued Approximation. Journal of Approximation Theory, 91, 17-92. https://doi.org/10.1006/jath.1993.1030

[5] Asiry, M. and Watson, G.A. (1999) On Solution of a Class of Best Simultaneous Approximation Problems. International Journal of Computer Mathematics, 75, 413-425. https://doi.org/10.1080/00207169908804818

[6] Asiry, M. and Watson, G.A. (2000) Simultaneous Approximation from Chebyshev and Weak Chebyshev Spaces. Communications in Applied Analysis, 4, No. 3. 
[7] Alyazidi-Asiry, M. (2016) Adjoining a Constant Function to N-Dimensional Chebyshev Space. Journal of Function Spaces, 2016, Article ID: 4813979. https://doi.org/10.1155/2016/4813979

[8] Alyazidi-Asiry, M. (2017) Extending a Chebyshev Subspace to a Weak Chebyshe Subspace of Higher Dimension and Related Results. Journal of Applied and Computational Mathematics, 6, Article ID: 1000347. https://doi.org/10.4172/2168-9679.1000347

[9] Cheney, E.W. (1966) Introduction to Approximation Theory. McGraw-Hill, New York, London.

[10] Watson, G.A. (1980) Approximation Theory and Numerical Methods. John Wiley \& Sons, Chichester. 